SIMPOSIO: GÉNERO Y SALUD

\title{
EL ROL DE LAS REDES SOCIALES Y EL EMPODERAMIENTO DE LAS MUJERES EN MEDICINA
}

\author{
Elizabeth Espinoza-Portilla $\mathbb{1}^{1, a}$, Victor J. Linares-Cabrera ${ }^{2, b}$ \\ 1 Universidad ESAN, Lima, Perú. \\ 2 Universidad Nacional José Faustino Sánchez Carrión, Huacho, Perú. \\ a Médica cirujana, doctora en Gobierno y Políticas Públicas; ${ }^{\text {b }}$ economista, magíster en Docencia Superior e \\ Investigación Universitaria.
}

\section{RESUMEN}

Hoy en día la cantidad de médicos y profesionales de salud que utilizan las redes sociales como vehículos principales para la comunicación está en aumento. Las redes sociales ofrecen nuevas formas de conectarse, facilitan la comunicación casi instantánea sin límites geográficos y sirven de apoyo a estudiantes, médicos y profesionales de salud. Las redes sociales pueden permitir que las mujeres en el campo de la salud obtengan apoyo que de otro modo no tendrían. Este artículo describe el rol de las redes sociales para contribuir al empoderamiento de las mujeres en medicina y discute las oportunidades de utilizar las redes sociales como herramienta para acceder a mentoría y consejeros experimentados como modelos a seguir; el apoyo e interacción entre pares; y el acceso a información y educación continua.

Palabras clave: Redes Sociales; Mujeres; Médicos Mujeres; Envío de Mensajes por Twitter; Perú (fuente: DeCS BIREME).

\section{THE ROLE OF SOCIAL NETWORKS AND WOMEN EMPOWERMENT IN MEDICINE}

\begin{abstract}
Today, the number of doctors and health professionals who use social networks as main venues for communication is increasing. Social networks offer new ways to connect and can facilitate almost instantaneous communication without geographical boundaries and can support students, doctors and health professionals. Social networks can allow women in the health field to obtain support that they would not otherwise have. This article describes the role of social networks in contributing to the empowerment of women in medicine and discusses the opportunities to use social networks as a tool for: access to mentoring and experienced counselors as role models; peer support and interaction; and access to information and continuing education.
\end{abstract}

Keywords: Social Networking, Women, Women Physicians, Twitter Messaging, Peru (source: MeSH NLM).

\section{INTRODUCCIÓN}

Citar como: Espinoza-Portilla E, Linares-Cabrera VJ. El rol de las redes sociales y el empoderamiento de las mujeres en medicina. Rev Peru Med Exp Salud Publica. 2019;36(2):13641. Doi: https://doi.org/10.17843/ rpmesp.2020.371.5092

Correspondencia: Elizabeth Espinoza-Portilla. Correo electrónico: eespinozap@esan.edu.pe San Borja Sur 441, San Borja, Lima

Recibido: $10 / 01 / 2020$ Aprobado: $11 / 03 / 2020$ En línea: 23/03/2020
La Agenda 2030 de los objetivos de desarrollo sostenible de las Naciones Unidas establece la participación y el liderazgo de las mujeres como fundamentales en todos los ámbitos del desarrollo $^{(1)}$. En las últimas décadas, se ha manifestado una creciente participación de las mujeres en puestos profesionales en medicina, particularmente tuvo un incremento sistemático y muy significativo desde la década de los $80^{(1)}$. Lograr una mayor participación femenina en el campo de la medicina es una realidad que debemos estudiar, comprender y fomentar ${ }^{(2)}$.

Sin embargo, las médicas - y de manera similar el personal técnico femenino y las profesionales de la salud en general- enfrentan una serie de barreras y desafíos que se manifiestan desde el inicio de su formación. Las mujeres que abandonan sus estudios académicos luego de comenzar sus carreras manifiestan diversos factores asociados, como la desigualdad salarial, la falta de modelos a seguir, pocas oportunidades de reconocimiento, dificultades para lograr una adecuada inserción laboral, poca presencia en posiciones de liderazgo, menor participación femenina en investigación, incluyendo brechas de financiamiento, falta de redes de colaboración, y ambientes de trabajo no apropiados ${ }^{(3,4)}$. Incluso se ha reportado que las médicas 
y científicas reciben un trato dispar en los eventos y conferencias, es decir, pueden ser invitadas a dar una conferencia, pero no la charla magistral ni las ponencias principales, lo cual afecta negativamente sus líneas de carrera ${ }^{(5,6)}$.

En el campo médico universitario, las médicas perciben salarios más bajos, comparados con los médicos, y las investigadoras reciben menos fondos de organizaciones que promueven la investigación, tienen menos oportunidades de publicar y son promovidas a tasas más lentas y más bajas ${ }^{(7)}$. Aunque se están desarrollando iniciativas a nivel nacional y mundial para ayudar a avanzar y promover a las mujeres a puestos de liderazgo, estas preocupaciones persisten para las mujeres que intentan ascender a posiciones más altas en el ámbito médico y universitario ${ }^{(8)}$. Por ello, las mujeres deben considerar otras estrategias y enfoques complementarios para obtener las habilidades necesarias para impulsar su vida profesional.

Por otro lado, hoy en día la cantidad de médicos y profesionales de salud en general que utilizan las redes sociales como vehículos principales para la comunicación está en aumento ${ }^{(8)}$. Las redes sociales ofrecen nuevas formas de conectarse y pueden facilitar la comunicación casi instantánea sin límites geográficos y pueden servir de apoyo a estudiantes, médicos y profesionales de salud ${ }^{(9-11)}$.

En este contexto, las médicas y profesionales de salud han revelado a través de las redes sociales muchos aspectos de su vida profesional, incluyendo particularidades de su vida personal y profesional ${ }^{(8)}$. Sus discusiones virtuales han logrado concientizar sobre cuestiones relacionadas con el empoderamiento de las mujeres en medicina y en la salud ${ }^{(8)}$. Incluso las redes sociales podrían permitir que las mujeres en el campo de la salud obtengan apoyo que de otro modo no tendrían.

Este artículo describe el rol de las redes sociales para contribuir al empoderamiento de las mujeres en medicina y discute las oportunidades de utilizar estas redes como herramienta para acceder a mentoría y consejeros experimentados como modelos a seguir; el apoyo e interacción entre pares; y el acceso a información y educación continua en el campo de la salud.

\section{EVOLUCIÓN DE LA PARTICIPACIÓN FEMENINA EN EL CAMPO DE LA MEDICINA}

Históricamente se ha evidenciado un rezago en la participación femenina en el campo de la medicina a nivel mundial y también en nuestro país ${ }^{(2)}$. En los primeros 50 años desde que las mujeres ingresaron a estudiar medicina en el Perú, solo se tuvo a cuatro médicas ${ }^{(2)}$. Sin embargo, en las últimas décadas el interés de las mujeres por estudiar medicina, y otras carreras universitarias, se ha incrementado ${ }^{(2)}$. Según el Instituto Nacional de Estadística e Informática del Perú, las egresadas universitarias representaron más de la mitad $(56,6 \%)$ de la población de egresados universitarios entre 21 a 30 años a nivel nacional el $2014^{(12)}$.

Además, la proporción de mujeres médicas colegiadas en el Perú se ha cuadriplicado. Del 14,5\% de médicas colegiadas en $1975^{(2)}$ pasamos al 49,5\% el $2016^{(13)}$. Según Ramírez-Orellana y León Rivera ${ }^{(13)}$ al analizar los médicos colegiados registrados en el Colegio Médico del Perú durante el periodo 2007-2016, encontraron que entre 2007 y 2009 la relación de mujeres fue en aumento, siendo la razón hombre/mujer de 1:1,49 el 2009. Sin embargo, dicha relación descendió los siguientes años y no se volvió a repetir esta situación ${ }^{(13)}$.

Asimismo, existe una brecha en la participación femenina en la autoría de publicaciones médicas. Según García-Aguilar et al., al analizar los artículos de la Revista Peruana de Medicina Experimental y Salud Pública, encontró que solo el $27,8 \%$ tuvo a una mujer como primera autora ${ }^{(14)}$. Esta disparidad puede estar asociada a los desafíos que enfrentan las médicas y las mujeres que laboran en el campo de salud, como compatibilizar sus labores de cuidado de niños, familiares o conocidos en situación de dependencia, y de sí mismas, con sus carreras ${ }^{(15)}$. Estos desafíos pueden ser incluso más complejos cuando ocupan puestos subordinados durante el proceso de entrenamiento, por ejemplo la residencia, periodo en el que muchas mujeres guardan silencio ante comentarios machistas o degradables ${ }^{(16)}$.

\section{LAS REDES SOCIALES Y EL EMPODERAMIENTO DE LAS MUJERES}

Las redes sociales pueden brindar a las mujeres que laboran en el campo de la salud oportunidades que generaciones anteriores no tenían para expresar sus opiniones, ideas y visión sobre sus carreras y especialidades ${ }^{(8)}$. Las redes sociales ofrecen más oportunidades para establecer contactos y conocer personas y lugares. Dichas plataformas también pueden convertirse en canales de gran alcance para difundir sus trabajos e investigaciones, lo que, a su vez, puede incrementar las oportunidades para construir reputación profesional y diseminar sus portafolios académicos ${ }^{(8)}$.

Las redes sociales están surgiendo como plataformas para compartir, ayudar y elevar la voz de las mujeres cuando su voz está restringida, y el tópico de redes sociales y empoderamiento de las mujeres se está convirtiendo en una herramienta poderosa para tomar conciencia y acción sobre las circunstancias que pueden estar atravesando ${ }^{(17)}$.

El empoderamiento de las mujeres es necesario para un futuro brillante de las mujeres, la familia, la sociedad y el país, y para que estas tomen sus propias decisiones en su crecimiento personal ${ }^{(17)}$. Durante mucho tiempo, la voz de muchas mujeres ha estado restringida y ahora puede diseminarse a través de las redes sociales ${ }^{(17)}$. La presencia en redes 
sociales brinda a las mujeres una nueva forma de libertad, independencia, control, y empoderamiento como nunca antes ${ }^{(17)}$. En las redes sociales se plantean y responden preguntas y pueden servir para que el grupo brinde soporte tanto intelectual como emocional ${ }^{(17)}$. Todo ello genera un nuevo tipo de dinamismo y empoderamiento tecnológico.

\section{OPORTUNIDADES DE PARTICIPACIÓN EN LAS REDES SOCIALES}

A continuación, se discuten tres mecanismos prioritarios por medio de los cuales las redes sociales, y Twitter en particular, se pueden convertir en oportunidades para el desarrollo profesional de las médicas y las profesionales de la salud en general.

\section{Acceso a mentoría y consejeros experimentados como modelos a seguir}

La mentoría es importante porque un mentor o un consejero experimentado comparte conocimientos y experiencias que permiten desarrollar destrezas para guiar a su discípulo o mentorizado en el inicio de su camino dentro del ámbito científco profesional, en una relación basada en la consideración mutua (18). Identificar un modelo a seguir en un profesional experto que «se parezca a uno» facilita el proceso ${ }^{(7)}$. Sin embargo, programas formales de mentoría son poco frecuentes en el Perú ${ }^{(19)}$. En el mundo existen programas de mentoría en instituciones académicas para médicas y profesionales de la salud que inician sus carreras. En Estados Unidos, existen conferencias y seminarios de liderazgo para mujeres organizados por la Asociación Americana de Escuelas de Medicina (AAMC, por sus siglas en inglés), cuyas actividades pueden seguirse en su cuenta de Twitter (@AAMCProfDev), o grupos como la Asociación Americana de Mujeres Médicas (AMWA, por sus siglas en inglés) que comparten sus actividades a través de su cuenta de Twitter (@AMWAdoctors). Estas asociaciones se esfuerzan por conectar a las mujeres en ámbitos académicos de la medicina con apoyo, tutoría, y seminarios en línea ${ }^{(7)}$. Sin embargo, la capacidad de conexión para los mentores y mentorizados se encuentra limitada por factores como la distancia, el tiempo dedicado para la mentoría, y la poca cantidad de mentores ${ }^{(7)}$. Es fundamental que instituciones académicas y colegios profesionales promuevan cursos y programas de liderazgo para mujeres profesionales en el campo de la medicina y la salud, y que se complementen con el uso interactivo de las redes sociales, como Twitter ${ }^{(9-1)}$.

Los educadores también se han dado cuenta de la necesidad de recurrir a redes de aprendizaje paralelas para complementar información y han recurrido a Twitter y a otras plataformas sociales como extensiones del entorno de aprendizaje ${ }^{(20)}$. Las relaciones desarrolladas en Twitter pueden ayudar a llenar los vacíos de los métodos más tradicionales de enseñanza y mentoría en persona ${ }^{(21)}$. Para ello, una forma de entablar y buscar potenciales mentores y contactar con profesionales e investigadores se da a través de los hashtags o etiquetas que permiten ubicar palabras específicas sobre un tema y participar en discusiones con personas de todo el mundo en seminarios, conferencias y congresos ${ }^{(10)}$.

El valor de acceder a un gran grupo de profesionales que pueden convertirse en modelos a seguir, potenciales mentores y sobre todo siendo del mismo género en las redes como Twitter puede ser particularmente importante en situaciones en las que estas relaciones no se encuentran disponibles de manera uniforme para residentes médicas o profesoras jóvenes ${ }^{(7)}$. Por ejemplo, la cuenta en Twitter @ WomenInThoracic está orientada a médicas interesadas en la cirugía cardiaca y torácica, ofrece tutoría a cirujanas jóvenes y oportunidades educativas para cirujanas torácicas. Toda esta interacción y comunicación en comunidad promueve aprendizajes informales y contribuye positivamente a la creación de capital social ${ }^{(7)}$.

Además, en el tema de liderazgo, es necesario que las mujeres identifiquen y construyan relaciones con sus pares que las apoyen a construir y desarrollar un liderazgo propio ${ }^{(22)}$. Twitter proporciona un foro alternativo para que las profesionales de la salud se conecten más allá de los espacios tradicionalmente liderados por varones. Movimientos como \#HeforShe resaltan esta necesidad e identifican a aquellos que están dispuestos a participar en campañas de sensibilización generadas en las redes sociales ${ }^{(22)}$. Aunque es casi imposible que los mensajes de 280 caracteres disponibles en el Twitter constituyan una tutoría sustantiva, la plataforma prepara el escenario para que muchas profesionales conozcan y accedan a mentores o consejeros experimentados con los que de otro modo no tendrían la oportunidad de conectarse. Estas conexiones pueden entonces promover mentorías informales y formales para apoyar el desarrollo de las profesionales de la salud ${ }^{(8)}$.

\section{Apoyo e interacción entre pares}

Las médicas y profesionales de salud discuten una variedad de incidentes y problemas en las redes sociales, incluidos temas sensibles o personales que históricamente se habrían compartido solo de forma privada. Dado que las redes sociales están disponibles las 24 horas los siete días a la semana, las mujeres pueden conectarse a su conveniencia. Hace más de una década, Robinson describió los estereotipos de las médicas como «demasiado sensibles» si expresaban inquietudes sobre cuestiones que les eran exclusivas como la necesidad de contar con espacios para lactancia en eventos o conferencias médicas ${ }^{(23)}$. Robinson describió los factores estresantes específicos para las médicas ${ }^{(23)}$, incluyendo discriminación en el lugar de trabajo, la falta de modelos a seguir y mentores, remuneraciones más bajas y menos recursos que los que recibieron sus pares, y el conflicto de roles entre la maternidad y su vida profesional, temas que siguen siendo de mucha relevancia hoy en día. 
¿Qué temas se podrían abordar con la interacción entre pares en las redes sociales? Repasemos la situación por la que atraviesan muchos profesionales de la salud a nivel nacional y global. En 2016, Jagsi et al. encontró en una muestra de investigadores clínicos de la Universidad de Michigan (Estados Unidos), que el 30\% de las mujeres y el $4 \%$ de los hombres informaron haber sufrido acoso sexual ${ }^{(24)}$. Jagsi señaló que después de que se publicó el estudio, ninguna de las mujeres que habían experimentado acoso había denunciado esos incidentes a ningún líder de su organización ${ }^{(25)}$. Jaqsi menciona la influencia de culturas institucionales desafiantes, la cultura machista, con lugares de trabajo dominados por hombres que mantienen «conversaciones de vestuario» lascivas o que excluyen a médicas de eventos sociales masculinos, dejándolas sin aliados en los que puedan confiar después de sufrir una indignidad o alguna agresión ${ }^{(25)}$.

En Perú, de acuerdo a un estudio de Vidal Panduro et al. ${ }^{(26)}$ basado en los datos de la Encuesta Nacional de Satisfacción de Usuarios de Salud del 2016, se encontró que de 2216 médicos el 25,2\% (583) reportó haber sido víctima de al menos un hecho violento; $13,1 \%$ (353) de amenaza; $19,8 \%$ (462) de insulto; $2,1 \%$ (65) de agresión física; y 1,6\% (22) de acoso sexual en los últimos 12 meses. No diferencian en su análisis por sexo. En Lima, Taype-Rondán et al. ${ }^{(27)}$ encuestaron a médicos egresados de una universidad privada y encontraron que el $23,9 \%$ reportaron haber sufrido acoso sexual alguna vez durante su internado, de los cuales el 35,7\% identificó que el agresor fue un paciente o familiar, y el 64,3\% identificó que fue un profesional de la salud. Los autores encontraron que el acoso sexual fue mayor en mujeres, en menores de 25 años y en sedes que no fueron del Ministerio de Salud. Por su parte, Munayco-Guillén et al. ${ }^{(28)}$ aplicaron una encuesta en una muestra de estudiantes de medicina de Ica y encontraron que el tipo de maltrato más frecuente fue haber recibido más de una vez comentarios negativos o despectivos $(89,8 \%)$ y haber recibido críticas injustificadas (82,1\%).

Las redes sociales pueden ayudar a compartir estos tipos de experiencias, y se ha visto con otros tipos de acoso a la mujer. Por ejemplo las mujeres usan varios mecanismos de Twitter para encontrarse y establecer comunidades virtuales. El movimiento popularizado por Alyssa Milano a través de Twitter con el hashtag \#MeToo para que las mujeres dieran a conocer las violencias sexuales a las que se habían enfrentado se volvió viral y propició que mujeres de todo el mundo se animasen a testimoniar sus experiencias de acoso y violencia sexual ${ }^{(29)}$. Luego apareció el hashtag \#Yotambién para compartir experiencias similares en español. En el ámbito médico, usuarios en Twitter comenzaron a utilizar los hashtags \#MeTooMedicine, \#WomeninMedicine o incluso \#WomenInSTEM para compartir situaciones por las que atraviesan las colegas y conseguir apoyo en las redes sociales ${ }^{(8)}$. Las estudiantes de medicina a menudo usan el hashtag \#GirlMedTwitter, y las cirujanas, el hashtag \#ILookLikeASurgeon. Estos hashtags pueden usarse junto con otros relacionados con la especialidad (por ejemplo: \#diabetes) o pueden unirse en Twitter a grupos que apoyan a las mujeres médicas como @WomenSurgeons y @ womenMDinanesth para encontrar a colegas con ideas afines o similares con quienes compartir ideas. En español existe el hashtag \#MujeresEnMedicina y la cuenta @Mujeresenmed que difunde mensajes sobre liderazgo de las mujeres en medicina en la red y promueven mentoría, y @Medicinamujeres, espacio creado para difundir los logros de las mujeres en medicina.

Shillcutt y Silver consideran que las redes sociales pueden ofrecer a las médicas y profesionales de salud mecanismos adicionales para afrontar y proporcionar nuevas vías para compartir información y quizás reducir el estigma asociado con el acoso sexual, al burnout o agotamiento y a la cultura laboral, al permitir que las experiencias se compartan y validen, disminuyendo el aislamiento social y los sentimientos de soledad o incluso de autoculpa ${ }^{(8)}$.

Los grupos en línea enfocados para médicas en español aún son limitados, pero estimamos que se incrementarán en el futuro, ya que proporcionan soporte para superar las barreras y superar los obstáculos para el desarrollo profesional, incluyendo la discriminación de género. A medida que las médicas formen comunidades con profesionales afines que compartan experiencias similares, será importante investigar la capacidad de dichas comunidades para disminuir o eliminar las barreras de género en la medicina.

\section{Acceso a información y educación continua}

Las plataformas como Twitter también se utilizan para comunicar mensajes educativos clave sobre investigación u otros temas ${ }^{(9)}$. El uso de Twitter por parte de los médicos ha crecido dramáticamente en los últimos años como un medio para promover la educación y vincular a los médicos con intereses comunes ${ }^{(10-11)}$. Las médicas y profesionales de salud pueden compartir en Twitter noticias e investigaciones que resulten relevantes para la comunidad, y con ello, las redes pueden servir de apoyo para construir y fortalecer su reputación profesional y promover sus carreras académicas ${ }^{(9)}$.

La difusión de investigaciones en las redes sociales puede ser especialmente beneficiosa para las mujeres, ya que no depende de la tutoría ni de las invitaciones a conferencias, áreas en las que las mujeres tienden a estar en desventaja ${ }^{(10-11)}$. Las mujeres que publican en revistas biomédicas pueden conseguir comentarios o nuevas ideas sobre sus trabajos de investigación a través de métricas alternativas, que están creciendo en importancia. Para incrementar el impacto de las publicaciones es recomendable incluir en el mensaje de Twitter el identificador «DOI» del artículo de la revista, para poder rastrear el alcance del artículo en línea. Muchas revistas especializadas comparten alertas y las publicaciones de sus números incluyendo los simposios a través de sus cuentas de Twitter, tal es el caso de la Revista Peruana de Medicina Experimental y Salud Pública, a través de su cuenta @rpmesp. Cabe resaltar que el 2016 la Clínica Mayo (Estados 
Unidos) se convirtió en uno de los primeros centros médicos académicos en incluir el uso de las redes sociales dentro de los criterios de promoción para sus profesionales, tomando como referencia unas directrices que pueden ser consideradas por instituciones peruanas para fomentar e incentivar el uso de las redes sociales en los profesionales de la salud ${ }^{(8)}$.

\section{CONCLUSIONES Y RECOMENDACIONES}

Los médicos y profesionales de la salud de todas las edades están utilizando las tecnologías de información y comunicación ${ }^{(30)}$, incluyendo las redes sociales; y muchas mujeres se están comunicando en plataformas virtuales para conectarse entre sí y apoyarse. La gran cantidad de mujeres médicas que participan y su compromiso sugieren que se valoran estas conexiones en línea. Sin embargo, hay que tomar en cuenta que para las médicas puede existir un riesgo reputacional involucrado en apoyar públicamente la equidad de género u otros esfuerzos de diversidad e inclusión ${ }^{(8)}$. Asimismo, es importante recordar que el comportamiento en redes sociales debe ser siempre profesional. Como menciona Curioso ${ }^{(9)}$ : «El respeto a la privacidad y al buen nombre, y el comportamiento deontológicamente correcto se vuelven obligaciones constantes en las redes sociales. Así no existan normas o disposiciones legales que lo obliguen. Esto es una manifestación de auto-regulación o profesionalismo».

Debemos considerar que, para bien o para mal, las redes sociales también registran de manera permanente el comportamiento no profesional. Por ello, es importante evitar la publicación de mensajes amenazadores, obscenos, hirientes, abusivos, vergonzosos o comentarios difamatorios ${ }^{(9)}$. Es

\section{REFERENCIAS BIBLIOGRÁFICAS}

1. Programa de las Naciones Unidas para el Desarrollo. Género en el Sector Salud: Feminización y brechas laborales [Internet]. Buenos Aires: PNUD; 2018 [citado el 2 de enero de 2020]. Disponible en: https://www.undp. org/content/dam/argentina/Publications/Desarrollo\%20Humano/ PNUD_InformedeGenero_2018.04.04.pdf

2. Pamo-Reyna OG. Una visión histórica de la participación femenina en la profesión médica. Rev Soc Peru Med Interna 2007;20(3):109-122.

3. Barfield WL, Plank-Bazinet JL, Austin CJ. Advancement of Women in the Biomedical Workforce: Insights for Success. Acad Med. 2016;91(8):10479. doi: 10.1097/ACM.0000000000001274

4. Levine RB, Lin F, Kern DE, Wright SM, Carrese J. Stories from early-career women physicians who have left academic medicine: a qualitative study at a single institution. Acad Med. 2011;86(6):752-8. doi: 10.1097/ ACM.0b013e318217e83b

5. Boiko JR, Anderson AJM, Gordon RA. Representation of women among academic grand rounds speakers. JAMA Intern Med 2017;177(5):722-4. doi: 10.1001/jamainternmed.2016.9646

6. Klein RS, Voskuhl R, Segal BM, Dittel BN, Lane TE, Bethea JR, et al. Speaking out about gender imbalance in invited speakers improves diversity. Nat Immunol 2017;18:475-8. doi: 10.1038/ni.3707

7. Lewis JD, Fane KE, Ingraham AM, Khan A, Mills AM, Pitt SC, et al. Expanding Opportunities for Professional Development: Utilization of necesario también estar atento a los riesgos de intimidación en línea y el acoso cibernético a los que puedan estar expuestas particularmente el personal de salud femenino.

Es importante promover una cultura de usar Twitter adecuadamente entre los académicos. Las universidades incluso podrían promover el uso adecuado de plataformas sociales entre sus docentes e investigadores. Recomendamos a las universidades que cuentan con carreras relacionadas con las ciencias de la salud que diseñen e incluyan, en sus programas de formación, objetivos curriculares específicos para enseñar a los estudiantes, docentes, residentes, médicos asistentes e investigadores sobre el comportamiento profesional en las redes sociales. Los programas de capacitación deben incluir talleres sobre cómo sacar el máximo provecho de Twitter, facilitados por profesores experimentados en su uso. Cuando los docentes e investigadores publiquen artículos, se les debe alentar a compartirlos a través de Twitter. El uso de Twitter debe ser reconocido como parte de las actividades rutinarias de las actividades académicas (por ejemplo, en eventos y conferencias). Finalmente, se necesitan desarrollar estudios en nuestro país que evalúen si las redes sociales aportan al desarrollo profesional de las mujeres en los campos de la medicina y la salud.

Contribuciones de autoría: Todos los autores participaron en la concepción, diseño del trabajo revisión crítica y aprobación de la versión final. EEP realizó la redacción inicial del artículo. Todos los autores se hacen responsables de los aspectos que integran el manuscrito.

Fuentes de financiamiento: Autofinanciado.

Declaración de conflictos de interés: Los autores declaran no tener conflictos de interés en la publicación de este artículo.
Twitter by Early Career Women in Academic Medicine and Science. JMIR Med Educ. 2018;4(2):e11140. doi: 10.2196/11140

8. Shillcutt SK, Silver JK. Social Media and Advancement of Women Physicians. N Engl J Med. 2018;378(24):2342-2345. doi: 10.1056/NEJMms1801980

9. Curioso WH. Redes Sociales en Internet: Implicancias para estudiantes y profesionales en salud. Rev Med Hered. 2011;22(3):95-7.

10. Curioso WH, Alvarado-Vásquez E, Calderón-Anyosa R. Usando Twitter para promover la educación continua y la investigación en salud en el Perú. Rev Peru Med Exp Salud Publica. 2011;28(1):163-64. doi: 10.17843/ rpmesp.2011.281.478

11. Curioso WH, Carnero AM. Promoviendo la investigación en salud con Twitter. Rev Med Hered 2011;22(3):121-130.

12. Instituto Nacional de Estadística e Informática (INEI). Encuesta Nacional a Egresados Universitarios y Universidades, 2014 [Internet]. Lima: INEI; 2015 [citado el 2 de enero de 2020]. Disponible en: https://www.inei. gob.pe/media/MenuRecursivo/publicaciones_digitales/Est/Lib1298/ Libro.pdf

13. Ramírez-Orellana J, Leon-Rivera $H$. Tendencias en el número de médicos titulados anualmente en el Perú, 2007-2016: análisis de la variable género. Acta Med Peru. 2019;36(2):179-80

14. Garcia-Aguilar D, Heredia-Mimbela I, Pereyra-Elías R. Autoría femenina 
en la Revista Peruana de Medicina Experimental y Salud Pública: análisis del periodo 1997-2017. Rev Peru Med Exp Salud Publica. 2019;36(4):6019. doi: $10.17843 /$ rpmesp.2019.364.4488

15. Proaño Calderón A. Mujeres en el sector salud, ¿la balanza se inclina a su favor? [internet]. BID; 2019 [citado el 9 de enero del 2020]. Disponible en: https://blogs.iadb.org/salud/es/mujeres-en-salud/.

16. Kardashian A, May FP. Empowering early career female gastroenterologists and hepatologists. Nat Rev Gastroenterol Hepatol. 2019;16(11):644645. doi: 10.1038/s41575-019-0216-9

17. Vardhan R. Social media and women empowerment: a sociological analysis. International Journal of Economic and Business Review. 2017;5(6):117-121.

18. Caballero-Ortiz AG, Arroyo-Hernández H. Mentoría en la formación de investigadores. CIMEL 2014;19(1):3.

19. Lescano AG, Cohen CR, Raj T, Rispel L, Garcia PJ, Zunt JR, et al. Strengthening Mentoring in Low- and Middle-Income Countries to Advance Global Health Research: An Overview. Am J Trop Med Hyg. 2019;100(1_Suppl):3-8. doi: 10.4269/ajtmh.18-0556

20. Rehm M, Notten A. Twitter as an informal learning space for teachers!? The role of social capital in Twitter conversations among teachers. Teaching and Teacher Education. 2016;60:215-223. doi: 10.1016/j. tate.2016.08.015

21. Hart M, Stetten NE, Islam S, Pizarro K. Twitter and Public Health (Part 1): How Individual Public Health Professionals Use Twitter for Professional Development. JMIR Public Health Surveill. 2017 Sep 20;3(3):e60. doi: 10.2196/publichealth.6795

22. Pitt SC. What does \#NYerORCoverChallenge mean for men in cardiothoracic surgery? J Thorac Cardiovasc Surg. 2017;154(4):1352-1353. doi: 10.1016/j.jtcvs.2017.06.060
23. Robinson GE. Stresses on women physicians: consequences and coping techniques. Depress Anxiety. 2003;17(3):180-9. doi: 10.1002/da.10069

24. Jagsi R, Griffith KA, Jones R, Perumalswami CR, Ubel P, Stewart A. Sexual Harassment and Discrimination Experiences of Academic Medical Faculty. JAMA. 2016;315(19):2120-1. doi: 10.1001/jama.2016.2188

25. Jagsi R. Sexual Harassment in Medicine - \#MeToo. N Engl J Med. 2018;378(3):209-211. doi: 10.1056/NEJMp1715962

26. Vidal Panduro DA, Cochella Tizón OJ. Asociación entre violencia laboral externa e intención de migración en médicos que laboran en instituciones prestadoras de servicios de salud en Perú: análisis de la Encuesta Nacional de Satisfacción de Usuarios de los Servicios de Salud (ENSUSALUD 2016) [Internet].Lima, Perú: Universidad Peruana de Ciencias Aplicadas (UPC); 2019 [citado el 2 de enero de 2020]. Disponible en: https://repositorioacademico.upc.edu.pe/handle/10757/625800

27. Taype-Rondán Á, Lizarraga-Castañeda Z, Mayor-Vega A, Mariños-Claudet A. Percepción de violencia durante el internado de medicina en una universidad peruana. Rev Peru Med Exp Salud Pública. 2016;33(2):382-4. doi: 10.17843/rpmesp.2016.332.2188

28. Munayco-Guillén F, Cámara-Reyes A, Muñoz-Tafur LJ, Arroyo-Hernández $\mathrm{H}$, Mejia $\mathrm{CR}$, Lem-Arce $\mathrm{F}$, et al. Características del maltrato hacia estudiantes de medicina de una universidad pública del Perú. Rev Peru Med Exp Salud Publica. 2016;33(1):58-66. doi: 10.17843/ rpmesp.2016.331.2008

29. McQueen KAK. Women in Anesthesia. International Anesthesiology Clinics 2018;56(3):1-4. doi: 10.1097/AIA.0000000000000189

30. Curioso WH. Building Capacity and Training for Digital Health: Challenges and Opportunities in Latin America. J Med Internet Res. 2019;21(12):e16513. doi: 10.2196/16513 